\title{
Richtlinien «Feststellung des Todes mit Bezug auf Organtransplantationen»: wichtige Anpassungen
}

Die medizinisch-ethischen Richtlinien zur «Feststellung des Todes mit Bezug auf Organtransplantationen» waren die ersten Richtlinien, welche die Schweizerische Akademie der Medizinischen Wissenschaften veröffentlicht hat, und zwar 1969, also zehn Jahre vor der Gründung der Zentralen Ethikkommission. Seither wurden sie bereits zweimal überarbeitet, nämlich 1983 und 1996 (vgl. dazu unser Editorial in der Schweizerischen Ärztezeitung 2002;83[26]:1385). Die Fortschritte der Intensivmedizin und die damit verbundenen verbesserten Überlebenschancen machten es 2002 nötig, die Todeskriterien erneut anzupassen bzw. zu präzisieren. Ein Überleben ist dann ausgeschlossen, wenn die Funktionen des Gehirns irreversibel und vollständig ausgefallen sind und die Funktionen anderer Organe nur noch künstlich aufrechterhalten werden. Dabei kann der Ausfall des Gehirns verschiedene Ursachen haben: eine Verletzung des Gehirns aufgrund eines Unfalls, eine primäre Gehirnerkrankung oder einen länger dauernden Unterbruch der Gehirndurchblutung infolge eines Herz-Kreislauf-Stillstandes.

Der Entwurf zu den vorliegenden Richtlinien wurde im Jahre 2002 zur Vernehmlassung veröffentlicht; die eingegangenen Stellungnahmen wurden sorgfältig analysiert und wo möglich berücksichtigt. Die definitive Fassung wird in der heutigen Ausgabe der Schweizerischen Ärztezeitung veröffentlicht. Warum diese Verzögerung?

Das Bundesamt für Gesundheit hat die Akademie gebeten, die Richtlinien für den Bereich der Feststellung des Todes integral als Teil der Verordnungen zum neuen Transplantationsgesetz veröffentlichen zu können. Dies setzte voraus, dass sie mit dem Gesetzestext völlig im Einklang stehen, und erforderte mehrere Expertentreffen mit Vertretern des BAG und eine sorgfältige Überarbeitung des Textes. Der hauptsächliche Diskussionspunkt betraf die Festlegung des rechtsgültigen Todeszeitpunkts. Gemäss Gesetz kann dies nur der Moment der zweiten klinischen Untersuchung sein (T2), welche den Hirntod bestätigt. Demnach kann der Patient zwischen T1 und T2 nicht zweifelsfrei als tot betrachtet und muss daher entsprechend (d.h. als Lebender) behandelt werden. Grundsätzlich bestätigt die zweite Untersuchung nicht einfach die Resultate der ersten Untersuchung; durch den zeitlichen Abstand von mindestens 6 Stunden liefert sie gleichzeitig die Bestätigung, dass der Ausfall des
Gehirns irreversibel ist. Die Konsequenz dieser gesetzlichen Vorgabe besteht darin, dass zwischen T1 und T2 ohne vorgängige Einverständniserklärung des Spenders kein Eingriff zugunsten eines Dritten (d.h. des möglichen Empfängers) vorgenommen werden darf.

Die Frist von sechs Stunden und die zweite klinische Untersuchung werden jedoch hinfällig, wenn der Stillstand der Gehirndurchblutung, als eindeutiges und sicheres Zeichen des Gehirntodes, nachgewiesen werden kann. Im Vernehmlassungsentwurf war für diesen Nachweis einzig die zerebrale Angiographie als zuverlässige Standardmethode vorgesehen. Diese Untersuchung ist jedoch invasiv und mit Risiken behaftet und deshalb mit dem einzigen Ziel der Feststellung des Todes problematisch; aus diesem Grund sollten auch weniger eingreifende und genügend validierte Untersuchungsmethoden zur Verfügung stehen. In der Folge beauftragte die SAMW eine Expertengruppe mit Vertretern von Neurologie, Neuroradiologie, Intensivmedizin und Nuklearmedizin mit dieser Aufgabe; diese Gruppe traf sich zu mehreren Sitzungen (an der auch die Unterzeichneten teilnahmen), sichtete die Literatur und überprüfte die Praxis in anderen Ländern. Sie bezeichnete schliesslich aufgrund dieser Informationen und der eigenen Expertise vier zusätzliche Verfahren, die in die Richtlinie aufgenommen wurden; diese Liste kann in Zukunft regelmässig dem neusten Erkenntnisstand angepasst werden.

Der Senat der SAMW hat die definitive Fassung der Richtlinien an seiner Sitzung vom 24. Mai 2005 mit diesen Präzisierungen und Ergänzungen verabschiedet.

An dieser Stelle möchten wir allen, die an der Ausarbeitung dieser Richtlinien mitgewirkt haben - namentlich der verantwortlichen Subkommission und ihrem Präsidenten, Prof. Alex Mauron aus Genf, sowie den angefragten Experten und denjenigen, die sich im Rahmen der Vernehmlassung geäussert haben -, unseren Dank aussprechen. Ganz besonders danken möchten wir Prof. Werner Stauffacher, der als damaliger Präsident der SAMW an den Gesprächen mit den BAG-Vertretern teilgenommen und in der Folge die Entwicklung dieser Richtlinien eng begleitet hat.

Wie alle Richtlinien der SAMW können auch die vorliegenden auf der Website der SAMW (www.samw.ch) abgerufen werden. 